\title{
Absolute Stability of Single-Input Single-Output Systems with Constant Internal Point Time Delays
}

\author{
Manuel de la SEN \\ Instituto de Investigación y Desarrollo de Procesos, Facultad de Ciencias \\ Universidad del Pais Vasco \\ Leioa (Bizkaia), Aptdo, 644 de Bilbao, 48080-Bilbao, Spain \\ e-mail:msen@we.lc.ehu.es
}

Received: March 2003

\begin{abstract}
This paper deals with the absolute stability of single-input single-output time-delay systems with, in general, a finite number of non commensurate constant internal point delays for any nonlinearity satisfying a time positivity inequality related to the first and third quadrants. The results are obtained based on Lyapunov's stability analysis via appropriate Lyapunov's functions and the related stability study is performed to obtain both delay independent and delay dependent results.
\end{abstract}

Key words: absolute stability, dynamic systems, nonlinear feedback, time-delay systems.

\section{Introduction}

The absolute stability of dynamic is an interesting issue since it is referred to the global asymptotic stability of a system under any feedback law provided by a wide class of nonlinear devices. Such nonlinear devices have to satisfy a certain positivity sector-type constraints. The problem has been widely studied for the plant delay-free case and nonlinear feedback devices within linear sectors $\left[k_{1}, k_{2}\right]$ and $\left(k_{1}, k_{2}\right)$ in $(0, \infty)$. See, for instance, Vidyasagar (1993), Bergen (1967), Sen (1986), Gregor (1996), Sen (1998, 2002). Some of those results have been extended to single-delay cases provided that the transfer function of the linear subsystem is (non critically) stable (i.e., with poles in $\operatorname{Re} s<0$ ) provided that its $H_{\infty}$-norm is upper-bounded with a sufficiently small upper-bound and that the feedback nonlinear device satisfies certain local Liptschitzian regularity conditions, (Gorecki et al., 1989), and to systems with external delays (i.e., in the input), (Popov and Halanay, 1963). In this paper, such assumptions are removed by allowing nonlinearities simply satisfying a (in general non symmetric) sector-type positivity constraints, multiple non commensurate internal (i.e., in the state) delays and either strictly stable (the so-called Principal Case) or critically stable (the so-called Simplest Particular Case) linear plants with a single critically stable pole at $s=0$. The paper is organized as follows. Section 2 deals with the absolute stability independent of the sizes of the delays for all nonlinearities belonging to a sector $[0, \infty)$, what means simply that the nonlinearity output is constrained to the first/third quadrants being zero if and only if the plant output is zero. The system may be either in the Principal Case (or Direct Control); i.e., the linear 
plant in the forward loop is strictly stable or in the Simplest Particular Case (or Indirect Control); i.e., the linear plant possess a single critically stable pole at $s=0$. These points are discussed in the systems dealt with in Section 3. The above results are extended to stability dependent of the sizes of the delays in Section 4. Section 5 extends the problem of absolute stability to a new class of time-differentiable nonlinearities which do not necessarily belong to a sector in the first/third quadrants. Some examples are given in Section 6 and, finally, conclusions end the paper.

\subsection{Notation}

- The sets $\mathbf{R}, \mathbf{R}^{+}$and $\mathbf{R}_{\mathbf{0}}^{+}$denote, respectively, the sets of real numbers, positive real numbers and non negative real numbers.

- An output- feedback nonlinearity $\Phi(y(t))$ in a Popov's sector $\left[k_{1}, k_{2}\right] \subset[0, \infty)$ means that the scalar real function $\Phi: \mathbf{R} \times t[0, t] \rightarrow \mathbf{R}$ is such that $k_{1} y(t) \leqslant$ $\Phi(y(t)) \leqslant k_{2} y(t)$ for all $t \geqslant 0$ with $\Phi(y(t))=0$ if and only if $y(t)=0$.

- The Hardy space $R H_{\infty}$ of transfer functions or matrices G(s) are proper real rational functions with all its poles in $\operatorname{Re} \mathrm{s}<0$ ( i.e., strictly stable) and $H_{\infty}$-norm $\|G(s)\|_{\infty}=\operatorname{Sup}_{\omega \in \mathbf{R}_{0}^{+}}\left[\lambda_{\operatorname{Max}}^{1 / 2}\left(G^{T}(-j \omega) G(j \omega)\right)\right]$ with $\mathbf{R}_{\mathbf{0}}^{+}=\mathbf{R}^{+} \cup\{0\}$ and $\lambda_{\operatorname{Max}}($. being the maximum eigenvalue of the (.) - symmetric matrix.

- It is said that a transfer function $G(s)$ or matrix is strictly stable if $G(s) \in R H_{\infty}$ and its characteristic polynomial (or quasi-polynomial in the presence of internal delays) is strictly Hurwitzian.

- A linear transfer function $G(s)$ is in the principal case if it belongs to $R H_{\infty}$ and its characteristic polynomial (or quasi-polynomial in the presence of internal delays) is strictly Hurwitzian. It is in the simplest particular case if $G(s)=\frac{G_{0}(s)}{s}$ with $G_{0}(s) \in R H_{\infty}$.

- An unforced linear system with $r$ finite internal point delays $h_{i}$ of state equation $\dot{x}(t)=A x(t)+\sum_{i=1}^{r} A_{i} x\left(t-h_{i}\right)$ has two associate systems without delays, namely: $\dot{z}_{1}(t)=\left(A+\sum_{i=1}^{r} A_{i}\right) z_{1}(t)$ which describes the above so-called current delay-free system time-delay system when $h_{i}=0 ; i=\overline{1, r}$; and $\dot{z}_{2}(t)=A z_{2}(t)$ which is called the nominal delay-free system which describes the above time-delay system when $A_{i}=0$, or when $h_{i} \rightarrow \infty ; i=\overline{1, r}$.

Both systems have to be stable in order that the delay system be stable independent of the delays.

- The $l_{2}$-norm of a matrix (or vector ) $M$ is denoted as $\|M\|_{2}=\lambda_{\operatorname{Max}}^{1 / 2}\left(M^{T} M\right)$. In vectors such a norm coincides with the Euclidean norm. A positive definite (semidefinite) matrix $M$ is denoted as $M>0(M \geqslant 0)$. A negative definite (semidefinite) matrix $M$ is denoted as $M<0(M \leqslant 0)$. 


\section{Descriptions of Time-Delay Systems under Sector-Type Nonlinear Feedback}

Consider the single-input single-output linear and time-invariant system

$$
\begin{aligned}
& \dot{x}(t)=A x(t)+\sum_{i=1}^{r} A_{i} x\left(t-h_{i}\right)+b u(t), \\
& y(t)=c^{T} x(t)+d \xi(t)
\end{aligned}
$$

under one of the two nonlinear output-feedback control laws below:

$$
u(t)=\xi(t)=-\Phi(y(t))-\sum_{i=1}^{r} k_{i} \Phi\left(y\left(t-h_{i}\right)\right)
$$

or

$$
u(t)=\dot{\xi}(t)=-\Phi(y(t))-\sum_{i=1}^{r} k_{i} \Phi\left(y\left(t-h_{i}\right)\right),
$$

where $x(t) \in \mathbf{R}^{\mathbf{n}}, u(t) \in \mathbf{R}, y(t) \in \mathbf{R}$ are the state, input and output, respectively, and $A, A_{i} ; i=\overline{1, r}$, are real square $n$-matrices, $b, c \in \mathbf{R}^{\mathbf{n}}, d(\geqslant 0) \in \mathbf{R}$ and $k_{i} ; i=\overline{1, r}$ are real scalar gains. The initial condition of (1a) is any absolutely continuous function $\varphi:[-h, 0] \rightarrow \mathbf{R}^{\mathbf{n}}$ plus, eventually, a function of zero measure of isolated bounded discontinuities defined on $[-h, 0]$ where $h=\underset{1 \leqslant i \leqslant r}{\operatorname{Max}}\left(h_{i}\right)$. The nonlinear feedback device is defined via (1c) or (1d) by a nonlinear function $\Phi: \mathbf{R} \times[0, t] \rightarrow \mathbf{R}$ satisfying $\Phi(y)=0$ if and only if $\mathrm{y}=0$ and $0 \leqslant \Phi(y) \leqslant k$. This property is abbreviated referred to as $\Phi \in[0, k]$ or $\Phi(\mathrm{t})$ belongs to the sector $[0, k]$. In the same way, $0 \leqslant \Phi(y)<k$ is abbreviated to as $\Phi \in[0, k)$ or $\Phi(\mathrm{t})$ belongs to the sector $[0, k)$. The paper main results are concerned with the case when the sector upper-bound $\mathrm{k}$ is infinity. The configuration (1a)-(1c) is called the Principal Case and (1a)-(1b) and (1d) is called the Simplest Particular Case, both satisfying that the roots of $\operatorname{Det}\left(s I-A-\sum_{i=1}^{r} A_{i} \mathrm{e}^{-\mathrm{h}_{\mathrm{i}} \mathrm{s}}\right)=0$ implies $\operatorname{Re} \mathrm{s}<0$ so that the closed-loop system is globally asymptotically stable. In the Simplest Particular Case, the linear device adds an open-loop critically stable simple pole at $s=0$. The control law (1c) of the Principal Case (in Western terminology) is also called a Direct Control (Lure's /Popov's terminology) while (1d) being the control law of the Simplest Particular Case in Western terminology is also called Indirect Control (Lure's /Popov's terminology). The standard problem of absolute stability is that of global Lyapunov's stability for all nonlinearities of the given class when independent of the delays; i.e., for when $A_{i}=0 ; k_{i}=0 ; i=\overline{1, r}$ for all nonlinearities of the given class in a certain sector included in the first and third quadrants. In this paper, non zero gains $k_{i} ; i=\overline{1, r}$ in certain ranges are admitted as well as nonzero delays $h_{i}$ belonging to certain ranges may also be present; i.e., absolute stability dependent of the delays or for all $h_{i} \in[0, \infty)$; i.e., absolute stability independent of the delays. The second topic is addressed in Section 3 while the first one is addressed in Section 4. 


\section{Absolute Stability Independent of the Delays}

Consider the Lyapunov's function candidate:

$$
\begin{aligned}
V\left(x_{t}\right)= & x^{T}(t) P x(t)+\sum_{i=1}^{r} \int_{-h_{i}}^{0} x^{T}(t+\tau) S_{i} x(t+\tau) \mathrm{d} \tau+\mathrm{d} \xi^{2}(t) \\
& +2 q \int_{0}^{y} \Phi(\sigma) \mathrm{d} \sigma+\sum_{i=1}^{r} \int_{-h_{i}}^{0} q_{i} \Phi^{2}(y(t+\tau)) \mathrm{d} \tau
\end{aligned}
$$

where $P=P^{T}>0, S_{i}=S_{i}^{T}>0(i=\overline{1, r})$ are positive definite real $n$-matrices, $q>0, q_{i}>0(i=\overline{1, r})$ are real constants. The following result is proved in Appendix A.

Theorem 1. Assume (1) in the Simplest Particular Case and that there exist real $n$ matrices $P=P^{T}>0, S_{i}=S_{i}^{T}>0(i=\overline{1, r})$, and $l \in \mathbf{R}^{\mathbf{n}}, w \in \mathbf{R}, q \in \mathbf{R}^{+}$ and $\varepsilon \in \mathbf{R}^{+}$which satisfy the following three identities:

$$
\begin{aligned}
& A^{T} P+P A+\sum_{i=1}^{r} S_{i}=-\varepsilon P-l l^{T}, \\
& \left(I+A^{T} q\right)^{T} c-P b-w l=0, \\
& w^{2}=q\left(c^{T} b+d\right),
\end{aligned}
$$

and the matrix

$$
Q=\left[\begin{array}{cccccccc}
-\varepsilon P & P A_{1} & \ldots & P A_{r} & 0 & m_{1} & \ldots & m_{r} \\
A_{1}^{T} P & -S_{1} 0 & \ldots & 0 & q A_{1}^{T} c & 0 & \ldots & 0 \\
: & 0 \ldots & : & : & : & : & : & \\
A_{r}^{T} P & 0 & \ldots & -S_{r} & q A_{r}^{T} c & 0 & 0 & 0 \\
0 & q c^{T} A_{1} & \ldots & q c^{T} A_{r} & -q_{0} & -q_{1}^{\prime} & \ldots & -q_{r}^{\prime} \\
m_{1}^{T} & 0 & \ldots & 0 & -q_{1}^{\prime} & -q_{1} & \ldots & 0 \\
: & : & \ldots & : & : & : & : & : \\
m_{r}^{T} & 0 & \ldots & 0 & -q_{r}^{\prime} & 0 & \ldots & -q_{r}
\end{array}\right]
$$

where

$$
\begin{aligned}
& m_{i}=k_{i}(c-P b) ; \quad q_{i}^{\prime}=q\left(c^{T} b+d k_{i}\right)(i=\overline{1, r}), \\
& q_{0}=2 q\left(d+c^{T} b\right)-\sum_{i=1}^{r} q_{i} .
\end{aligned}
$$

Thus, the system (1) is absolutely stable independent of the delays for a nonlinear feedback law (1d), obtained from any nonlinear function $\Phi(y(t))$ in the sector $[0, \infty)$, 
so that $\left(x^{T}(t), \xi(t)\right)^{T}$ is bounded and converges asymptotically to zero as time tends to infinity, provided that $d \geqslant 0$ and $\left(d+c^{T} b\right)>0$.

Corollary 1. If (1) is in the Principal Case then it is absolutely stable independent of the delays for a feedback law (1c) from any nonlinear function $\Phi(y(t))$ in the sector $[0, \infty)$, if at least one of the two following sets of constraints hold:

1) (3)-(4) hold for the particular case $d=0$ and $c^{T} b>0$;

2) $c=P b, d \geqslant 0, d+c^{T} b>0$, (3a) holds with $q=0$ and $l=0$ (as a result (3b)-(3c) hold with $w=0$ and $l=0$ ) and

$$
Q^{\prime}=\left[\begin{array}{cccc}
-\varepsilon P & P A_{1} & \ldots & P A_{r} \\
A_{1}^{T} P & -S_{1} 0 & \ldots & 0 \\
: & 0 & \ldots & : \\
A_{r}^{T} P & 0 & \ldots & -S_{r}
\end{array}\right]<0
$$

Note that (3a) is a matrix Lyapunov's equation for the nominal delay-free system so that $A$ is a stability matrix so that all its eigenvalues are in $\operatorname{Re} s<0$. If (1a)-(1b) is a minimal state-space realization of dimension $n$ of a given transfer function then a similar result to Theorem 1 holds directly, but with dependence of the sizes of the delays, for any other non minimal realization $\left(A^{\prime}, b_{1}, c_{1}, d\right)$ of dimension $n_{1}>n$ for certain delay intervals $h_{i} \in\left[0, \bar{h}_{i}\right)$ provided that the pairs $\left(A^{\prime}, b_{1}\right)$ and $\left(c_{1}^{T}, A^{\prime}\right)$ are, respectively, stabilizable and detectable. This feature is obvious since there are $\left(n_{1}-n\right)$ stable zero-pole cancellations in the transfer function of the nominal delay-free system of such a realization apart from the $\mathrm{n}$ uncancelled poles, eigenvalues of $A$ and $A^{\prime}$, which are guaranteed to be stable from (3a). Note that the above stabilizability and detectability properties are associated with the corresponding properties of the state-space realizations of the nominal delayfree system of (1) for delays tending to infinity. The conclusion follows as a result of the continuity of the eigenvalues of the matrix of the dynamics (namely, $A+\sum_{i=1}^{r} A_{i} \mathrm{e}^{-\mathrm{h}_{\mathrm{i}} \mathrm{s}}$ ) with respect to the delays.

REMARK 1. Note that (4) holds by defining $Q=Q_{0}+\Delta Q$ if

$$
\Delta Q<-Q_{0}=-\operatorname{Block} \operatorname{Diag}\left(\varepsilon P, S_{1}, \ldots, S_{r}, q\left(c^{T} b+d\right)-\sum_{i=1}^{r} q_{i}, q_{1}, q_{2}, \ldots, q_{r}\right)<0 .
$$

The constraint (7) may be checked in terms of matrix norms as follows. Note from (3a) that

$$
P=\int_{0}^{\infty} \mathrm{e}^{\left(A^{T}+\varepsilon I\right) \tau}\left(l l^{T}+\sum_{i=1}^{r} S_{i}\right) e^{(A+\varepsilon I) \tau} \mathrm{d} \tau,
$$

where

$$
\|P\|_{2}=\lambda_{\max }(P) \leqslant \frac{K^{2}}{2 \rho}\left(\left\|l l^{T}+\sum_{i=1}^{r} S_{i}\right\|_{2}\right) \leqslant p_{1}=\frac{K^{2}}{2 \rho}\left(l^{T} l+\lambda_{\max }\left(S_{i}\right)\right)
$$




$$
\leqslant p_{2}=\frac{K^{2}}{2 \rho}\left(l^{T} l+r \operatorname{Max}_{1 \leqslant i \leqslant r}\left(\lambda_{\max }\left(S_{i}\right)\right)\right)
$$

and $\lambda_{\min }(P) \geqslant p_{01}=\frac{K^{2}}{2 \rho_{0}} r \operatorname{Min}_{1 \leqslant i \leqslant r}\left(\lambda_{\max }\left(S_{i}\right)\right)$ for some real constants $K \geqslant 1, \rho>$ $0, \rho_{0}>0$ such $\left\|\mathrm{e}^{(\mathrm{A}+\varepsilon \mathrm{I}) \mathrm{t}}\right\|_{2} \leqslant K \mathrm{e}^{-\rho \mathrm{t}}$. Thus, (7) holds using $p_{1}$ if

$$
\begin{aligned}
\|\Delta Q\|_{2} \leqslant \beta_{1} & =2\left[\sum_{i=1}^{r}\left\|A_{i}\right\|_{2}+\|b\|_{2} \sum_{i=1}^{r}\left|k_{i}\right| p_{1}+\left(q \sum_{i=1}^{r}\left\|A_{i}\right\|_{2}+\sum_{i=1}^{r}\left|k_{i}\right|\right)\|c\|_{2}\right. \\
& \left.+r q\left|c^{T} b\right|+d q \sum_{i=1}^{r}\left|k_{i}\right|\right]<\varepsilon p_{01} \leqslant \varepsilon \lambda_{\min }(P)
\end{aligned}
$$

if $\varepsilon$ is sufficiently small such that $\lambda_{\min }\left(Q_{0}\right)=\varepsilon \lambda_{\min }(P)$ for given $S_{i}, q_{i}(i=\overline{1, r})$ and $q$. It is also possible to state some refinements of the above condition as follows. An alternative condition may be derived after replacing $\beta_{1}$, calculating by using $p_{1}$ and $p_{01}$, with $\beta_{2}$, calculating in the same way by using $p_{2}$ and

$$
p_{02}=\operatorname{Min}\left(\frac{\varepsilon K r}{2 \rho_{0}} \operatorname{Min}\left(\lambda_{\min }\left(S_{i}\right)\right), \underset{1 \leqslant i \leqslant r}{\operatorname{Min}}\left(\lambda_{\min }\left(S_{i}\right)\right), q\left(c^{T} b+d\right)-\sum_{i=1}^{r} q_{i}, \underset{1 \leqslant i \leqslant r}{\operatorname{Min}}\left(q_{i}\right)\right) .
$$

Thus, $\Delta Q<-Q_{0}<0$ if $\left\|A_{i}\right\|_{2}$ and $\left|k_{i}\right|(\mathrm{i}=\overline{1, r})$ are sufficiently small such that $\beta_{1}<p_{01}$ or $\beta_{2}<p_{02}$ for some real constants $\varepsilon>0, q_{i}>0(i=\overline{1, r})$ and $q>\frac{\sum_{i=}^{R} q_{i}}{c^{T} b+d}>$ $0, n$-real matrices $S_{i}=S_{i}^{T}>0$, and a real vector $l=\frac{1}{\sqrt{q\left(c^{T} b+d\right)}}\left[\left(I+A^{T} q\right) c-P b\right]$ (from (3b)-(3c)) with $P=P^{T}>0$ defined in (8) (which then uniquely satisfies (3a)). Thus, it becomes obvious that Theorem 1 holds, as expected, if the absolute stability abscissa of the stability matrix $A$, defining the unforced dynamics of the nominal delayfree system, is sufficiently large and $\left\|A_{i}\right\|_{2}$ and $\left|k_{i}\right|(\mathrm{i}=\overline{1, r})$ are sufficiently small.

REMARK 2. Note that Theorem 1 is fulfilled if $Q \leqslant Q_{0}^{\prime}+\Delta Q^{\prime}$ fulfills $\Delta Q^{\prime}<-Q_{0}^{\prime}<0$ where

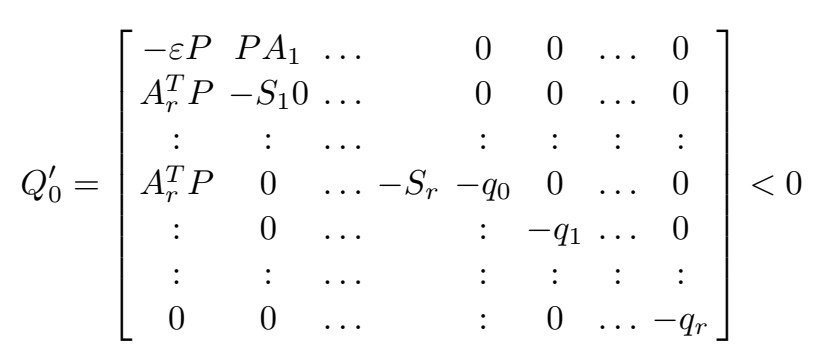




$$
\Delta Q^{\prime}=\left[\begin{array}{cccccccc}
0 & 0 & \ldots & 0 & 0 & m_{1} & \ldots & m_{r} \\
0 & 0 & \ldots & 0 & q A_{1}^{T} c & 0 & \ldots & 0 \\
: & 0 & \ldots & : & : & : & : & : \\
0 & 0 & \ldots & 0 & q A_{r}^{T} c & 0 & 0 & 0 \\
0 & q c^{T} A_{1} & \ldots & q c^{T} A_{r} & 0 & -q_{1}^{\prime} & \ldots & 0 \\
m_{1}^{T} & 0 & \ldots & 0 & -q_{1}^{\prime} & 0 & \ldots & 0 \\
: & : & \ldots & : & : & : & : & : \\
m_{r}^{T} & 0 & \ldots & 0 & -q_{r}^{\prime} & 0 & \ldots & 0
\end{array}\right]
$$

The above matrices are directly linked to a natural alternative expansion of $\dot{V}\left(x_{t}\right)$ used from (A.1) to prove Theorem 1 by replacing (A.2) by

$$
\begin{aligned}
\dot{V}\left(x_{t}\right) \leqslant & -\varepsilon x^{T}(t) P x(t)-\left(l^{T} x(t)-w \Phi\right)^{2}-q\left(c^{T} b+d\right) \Phi^{2} \\
& -2 \Phi y-\bar{x}^{T}(t) \Delta Q^{\prime} \bar{x}(t)<0,
\end{aligned}
$$

for all $\bar{x}(t) \neq 0$ and all $t \geqslant 0$.

It is interesting to link Theorem 1 and Corollary 1 with minimal state-space realizations of the nominal delay-free system associated with (1). In other words, it there are strictly stable uncontrollable and/or unobservable modes in the forward loop of the nominal delay-free system associated with (1), those ones may be removed from the transfer function of such a nominal delay-free system. As a result, the orders of the relevant matrices in testing Theorem 1/Corollary 1 may be reduced accordingly to the above mentioned strictly stable cancellations. Thus, consider a regular transformation defined by the nonsingular real $n$-matrix $T=\operatorname{Block} \operatorname{Diag}\left(T_{i j} ; i, j=1,2\right)$ to obtain an equivalent state-space realization to (1a), (1c) (i.e., a realization leading to identical transfer function) such that the dynamics matrix of the unforced delay-free nominal system takes a triangular form, which is defined by:

$$
\begin{aligned}
& A_{\Lambda}=T^{-1} A T=\left[\begin{array}{cc}
A_{0} & A^{\prime} \\
0 & A_{1}^{\prime}
\end{array}\right] ; \quad b_{\Lambda}=T^{-1} b=\left[\begin{array}{c}
b_{0} \\
b^{\prime}
\end{array}\right] ; \\
& A_{\Lambda i}=T^{-1} A T=\left[\begin{array}{cc}
A_{0 i} & A_{1 i}^{\prime} \\
0 & A_{2 i}^{\prime}
\end{array}\right] \quad(i=\overline{1, r}), \\
& c_{\Lambda}^{T}=c^{T} T^{-1}=\left[c_{0}^{T}, 0\right] ; d_{\Lambda}=d,
\end{aligned}
$$

with $\left(A_{0}, b_{0}\right)$ and $\left(c_{0}^{T}, A_{0}\right)$ being, respectively, a completely controllable pair and a completely observable pair. Both pairs are nontrivial in the sense that $b_{0}$ and $c_{0}$ are both non zero if the linear forward part of (1) is stabilizable and detectable. 
Corollary 2. Assume that $(A, b)$ is stabilizable, $\left(c^{T}, A\right)$ is detectable and $c_{0}=P_{0} b_{0}$ with $P_{0}=P_{0}^{T}>0$ being an $n_{0}(\leqslant n)$ real matrix satisfying:

$$
A_{0}^{T} P_{0}+P_{0} A_{0}+\sum_{i=1}^{r} S_{0 i}=-\varepsilon P_{0}-\frac{q}{c_{0}^{T} b_{0}+d} A_{0}^{T} P_{0} b_{0} b_{0}^{T} P_{0} A_{0},
$$

for some positive real constants $\varepsilon$ and $q$ and $n_{0}$ - real matrices $S_{0 i}=S_{0 i}^{T}>0(i=\overline{1, r})$. Thus, $A_{1}^{\prime}$ is a stability matrix if $n_{0}<n$ and the pairs $\left(A_{0}, b_{0}\right)$ and $\left(c_{0}^{T}, A_{0}\right)$ are, respectively, controllable and observable. Furthermore, the transfer function $G(s)=$ $b_{0}^{T} P_{0}\left(s I-A_{0}\right)^{-1} b_{0}=c^{T}(s I-A)^{-1} b$ is positive real; i.e., $\operatorname{Re}(G(s)) \geqslant 0$ for $\operatorname{Re} s>0$ and $G_{1}(s)=G(s)+d$ is strictly positive real; i.e., $\operatorname{Re}\left(G_{1}(s)\right)>0$ for $\operatorname{Re} s \geqslant 0$, if $d>0$ and positive real if $d \geqslant 0$. In addition, if $Q_{\Lambda}<0$ with $Q_{\Lambda}$ being obtained from (4) with the replacements $A \rightarrow A_{0}, b \rightarrow b_{0}, c \rightarrow c_{0}, A_{i} \rightarrow A_{0 i}(i=\overline{1, r})$, where $A_{0}$ and $A_{0 i}(i=\overline{1, r})$ are the $(1,1)$ block matrices of the partitions in four block matrices of $A_{\Lambda}=T^{-1} A T$ and $A_{\Lambda i}=T^{-1} A_{i} T(i=\overline{1, r})$, respectively.

Proof. Firstly, note that $b_{0}^{T} P_{0}\left(s I-A_{0}\right)^{-1} b_{0}=c^{T}(s I-A)^{-1} b$ is positive real from direct calculus, the second expression including (not including) strictly stable zero/pole cancellations if $n_{0}<n\left(n_{0}=n\right)$ since $\left(A_{0}, b_{0}\right)$ and $\left(c_{0}^{T}, A_{0}\right)$ are, respectively, controllable and observable pairs while $(A, b)$ and $\left(c^{T}, A\right)$ are, respectively, stabilizable and detectable pairs so that $A_{1}^{\prime}$ is a stability matrix if $n_{0}<n$. Also, similar equations to (3) are satisfied with the replacements $P \rightarrow P_{0}, A \rightarrow A_{0}, b \rightarrow b_{0}, c \rightarrow c_{0}=$ $P_{0} b_{0}, A_{i} \rightarrow A_{0 i}(i=\overline{1, r})$. Thus, the proof follows directly from Theorem 1 since $c_{0}^{T} b_{0}=b_{0}^{T} P_{0} b_{0}+d>0$ if $d \geqslant 0$.

Note that $c^{T}(s I-A)^{-1} b$ includes stable cancellations with $c^{T}=\left[b_{0}^{T} P T_{11}, b_{0}^{T} P T_{12}\right]$ since unstable or critically stable zero/pole cancellations in $G(s)$ cannot exist since $(A, b)$ is stabilizable and $\left(c^{T}, A\right)$ is detectable.

\section{Absolute Stability Dependent of the Sizes of the Delays}

The Lyapunov's function candidate (2) is modified to:

$$
\begin{aligned}
V^{0}\left(x_{t}\right)= & V^{\prime}\left(x_{t}\right)+\sum_{i=1}^{r}\left\{\int_{-h_{i}}^{0}\left(\int_{t+\tau}^{t} x^{T}(\theta) S_{i 0} x(\theta) \mathrm{d} \theta\right) \mathrm{d} \tau\right. \\
& \left.+\sum_{j=1}^{r} \int_{-h_{i}-h_{j}}^{-h_{j}}\left(\int_{t+\tau}^{t} x^{T}(\theta) S_{i j} x(\theta) \mathrm{d} \theta\right) \mathrm{d} \tau\right\},
\end{aligned}
$$

with $V^{\prime}\left(x_{t}\right)=V\left(x_{t}\right)-\sum_{i=1}^{r} \int_{-h_{i}}^{0} x^{T}(t+\tau) S_{i j} x(t+\tau) \mathrm{d} \tau$ and $V\left(x_{t}\right)$ defined in (2) where $P=P^{T}>0, S_{i j}=S_{i j}^{T}>0(i=\overline{1, r} ; j=\overline{0, r})$ are real positive definite $n$ - 
matrices. Direct calculus performed with (12) to calculate $\dot{V}^{0}\left(x_{t}\right)$ yields the following result.

Theorem 2. Assume (1) in the Simplest Particular Case and that there exist constant real $n$-matrices $P=P^{T}>0, S_{i j}=S_{i j}^{T}>0(i=\overline{1, r} ; j=\overline{0, r})$, and $l \in \mathbf{R}^{\mathbf{n}}, w \in \mathbf{R}, q \in$ $\mathbf{R}^{+}$and $\varepsilon \in \mathbf{R}^{+}$which satisfy (3) with (3a) being modified as follows:

$$
\chi(P)+l l^{T}+\varepsilon P=0,
$$

where

$$
\chi(P)=\left(A+\sum_{i=1}^{r} A_{i}\right)^{T} P+P\left(A+\sum_{i=1}^{r} A_{i}\right)+\sum_{i=1}^{r} h_{i}^{0}\left(S_{i}+\sum_{j=1}^{r} S_{i j}\right),
$$

and $Q$ in (4) is replaced with

$$
\bar{Q}=\operatorname{Block} \operatorname{Diag}\left(\bar{Q}_{i j} ; i, j=\overline{1,3}\right)=\bar{Q}^{T}<0,
$$

where

$$
\begin{aligned}
& \bar{Q}_{11}=\left[\begin{array}{cccc}
-\varepsilon P & h_{1}^{0} P A_{1} M \ldots & h_{r}^{0} P A_{1} M \\
h_{1}^{0} M^{T} A_{1}^{T} P & -h_{1}^{0} R_{1} & \ldots & 0 \\
: & 0 & : & : \\
\vdots & : & : & 0 \\
h_{r}^{0} M^{T} A_{r}^{T} P & 0 & \ldots & -h_{r}^{0} R_{r}
\end{array}\right]<0, \\
& R_{k}=\operatorname{Block} \operatorname{Diag}\left(S_{k 0}, S_{k 1}, \ldots, S_{k r}\right) ; M=\operatorname{Block} \operatorname{Diag}\left(A, A_{1}, \ldots, A_{r}\right), \\
& \bar{Q}_{12}^{T}=\left[0, q c^{T} A_{1}, \ldots, q c^{T} A_{r}\right] \in \mathbf{R}^{\mathbf{1} \times(\mathbf{r}+\mathbf{1}) \mathbf{n}} ; \bar{Q}_{22}=q\left(c^{T} b+d\right)-\sum_{i=1}^{r} q_{i}, \\
& \bar{Q}_{13}=\left[\begin{array}{c}
k_{1}(P b-c) \ldots k_{r}(P b-c) \\
\ldots . . \\
0
\end{array}\right] \quad\left(\in \mathbf{R}^{(\mathbf{r}+\mathbf{1}) \mathbf{n} \times \mathbf{r}),}\right\} r n \\
& \bar{Q}_{23}^{T}=\left[q\left(c^{T} b+d k_{1}\right), \ldots, q\left(c^{T} b+d k_{r}\right)\right] \in \mathbf{R}^{\mathbf{1} \times \mathbf{r}} ; \bar{Q}_{33}=\operatorname{Diag}\left(-q_{1}, \ldots, q_{r}\right) .
\end{aligned}
$$

Thus, the system is absolutely stable for all $\Phi$ in $[0, \infty)$ and all delays $h_{i} \in$ $\left[0, h_{i}^{0}\right] ;(i=\overline{1, r})$.

Guideline of proof. Using (12)-(16), one gets $\dot{V}^{0}\left(x_{t}\right) \leqslant-\bar{x}^{T}(t) \bar{Q} x(t) \leqslant 0$ for all time, the above inequality being strict for all $\bar{x}(t) \neq 0$.

REMARK 3. Note that it is necessary that $\bar{A}=A+\sum_{i=1}^{r} A_{i}+\varepsilon I$ is a stability matrix with stability abscissa $\left(-\rho_{1}\right)=-\bar{\rho}+\varepsilon<0$ in order that $\bar{Q}<0$ where $(-\bar{\rho})<0$ is the stability abscissa of the stability matrix $\left(A+\sum_{i=1}^{r} A_{i}\right)$ of the current delay-free system 
(i.e., $h_{i}=0 ; i=\overline{1, r}$ ) which is then stable as expected. Thus, for some real constant $\bar{K} \geqslant 1$,

$$
\|P\|_{2} \leqslant \frac{\bar{K}^{2}}{2(\bar{\rho}-\varepsilon)}\left[\lambda_{\max }\left(h_{i}^{0}\left(S_{i}+\sum_{j=1}^{r} S_{i j}\right)\right)\right],
$$

since from (13)-(14),

$$
P=\int_{0}^{\infty} \mathrm{e}^{\overline{\mathrm{A}}^{\mathrm{T}} \tau} Q^{\prime} \mathrm{e}^{\overline{\mathrm{A}} \tau} \mathrm{d} \tau ; \mathrm{Q}^{\prime}=1 \mathrm{ll}^{\mathrm{T}}+\sum_{\mathrm{i}=1}^{\mathrm{r}} \mathrm{h}_{\mathrm{i}}^{0}\left(\mathrm{~S}_{\mathrm{i}}+\sum_{\mathrm{j}=1}^{\mathrm{r}} \mathrm{S}_{\mathrm{ij}}\right) .
$$

Thus, all the constraints of Remark 1 to ensure that $\bar{Q}_{11}<0$ and $\bar{Q}<0$ hold with the replacements:

$$
\begin{aligned}
& A_{i} \rightarrow \bar{A}_{i}=h_{i}^{0} A_{i} M=h_{0}^{i} A_{i}\left[A, A_{1}, \ldots, A_{r}\right], \\
& S_{i} \rightarrow h_{i}^{0} R_{i}=h_{0}^{i} \text { Block Diag }\left[S_{i 0}, S_{i 1}, \ldots, S_{i r}\right],
\end{aligned}
$$

what yield the norm replacements:

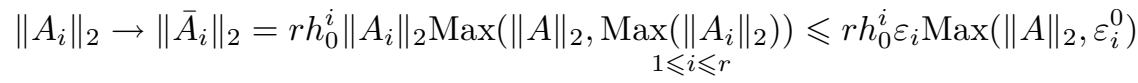

$$
\begin{aligned}
& \leqslant r h_{0}^{i} \varepsilon_{i}^{0}\left(a+\varepsilon_{i}^{0}\right) ; \quad \varepsilon_{i}^{0}=\operatorname{Max}_{1 \leqslant i \leqslant r}\left(\varepsilon_{i}\right), \\
& \left\|S_{i}\right\|_{2} \rightarrow h_{0}^{i}\left\|R_{i}\right\|_{2} \leqslant r h_{0}^{i} \underset{1 \leqslant j \leqslant r}{\operatorname{Max}}\left(\left\|S_{i j}\right\|_{2}\right) .
\end{aligned}
$$

Thus, for sufficiently small $\left\|A_{i}\right\|_{2},\left|k_{i}\right|(i=\overline{1, r}), \bar{Q}_{11}<0$ and $\bar{Q}<0$ so that depending on the set $h_{i}^{0}(i=\overline{1, r})$, the system (1) is absolutely stable on $[0, \infty)$ for $h_{i} \in\left[0, h_{i}^{0}\right],(i=\overline{1, r})$. Note, in particular, that if $A_{i}=0, h_{i}^{0}=k_{i}=0(i=\overline{1, r})$ then $x(t)$ is bounded and converges asymptotically to zero as time tends to infinity. That follows by choosing $q=q_{i}=0,(i=\overline{1, r})$ since the nominal delay-free system $\dot{z}(t)=\left(A+\sum_{i=1}^{r} A_{i}\right) z(t)$; i.e., that corresponding to $h_{i} \rightarrow \infty(i=\overline{1, r})$, is globally exponentially stable what is corroborated by the application of Theorem 2 to this particular case. The above result is also valid for the General Case in a natural way but the discussion is omitted.

REMARK 4. Corollary 2 may be directly extended in a natural way from Theorem 2 to the case of dependence with delays by replacing A with $\left(A+\sum_{i=1}^{r} A_{i}\right)$ and $A_{0}$ by the $(1,1)$ block matrix $\bar{A}_{0}=\left[T^{-1}\left(A+\sum_{i=1}^{r} A_{i}\right) T\right]_{11}$ resulting from a regular transformation $T$. Thus, by using (3b)-(3c), it follows that $b_{0}^{T} P_{0}\left(s I-\bar{A}_{0}\right)^{-1} b_{0}+d$ is positive real. 


\section{Extensions to Another Class of Nonlinear Devices}

Now, the feedback nonlinear function is considered to possess time-derivative for all time. For simplicity, it is assumed that $k_{i}=0 ; i=\overline{1, r}$. This results for the Simplest Particular Case yield:

$$
\begin{aligned}
& \dot{x}(t)=A x(t)+\sum_{i=1}^{r} A_{i} x\left(t-h_{i}\right)-b \Phi(y(t)), \\
& y(t)=c^{T} x(t)-d \Phi(y(t)) ; u(t)=\dot{\xi}(t)=-\Phi(y(t)) .
\end{aligned}
$$

It is assumed that for some appropriate time-varying gain $\mathrm{k}(\mathrm{t})$, the class of devices under consideration satisfies:

$$
\dot{\Phi}(y(t))=k(t) \dot{y}(t) ; \quad d \geqslant 0 .
$$

The combination of (17) yields:

$$
\dot{y}(t)=\frac{c^{T}\left(A x(t)+\sum_{i=1}^{r} A_{i} x\left(t-h_{i}\right)-b \Phi(t)\right)}{1+d k(t)},
$$

so that from $(17 \mathrm{c})-(18)$, one gets

$$
\dot{\Phi}(t)=\frac{k(t) c^{T}\left(A x(t)+\sum_{i=1}^{r} A_{i} x\left(t-h_{i}\right)-b \Phi(t)\right)}{1+d k(t)} .
$$

Consider the Lyapunov's function candidate:

$$
\begin{aligned}
V\left(x_{t}\right)= & x^{T}(t) P x(t)+x^{T}(t) \mathbf{q}_{\mathbf{0}} x(t)+\sum_{i=1}^{r} \int_{-h_{i}}^{0} x^{T}(t+\tau) S_{i} x(t+\tau) \mathrm{d} \tau+\mathrm{d} \xi^{2}(t) \\
& +q \int_{0}^{y} \Phi(\sigma) \mathrm{d} \sigma+q_{1} \Phi^{2}(y(t))+q_{2} \int_{0}^{y} \Phi(y(\tau)) y(\tau) \mathrm{d} \tau,
\end{aligned}
$$

for some real $n$-matrices $P=P^{T}>0, S_{i}=S_{i}^{T}>0, i=\overline{1, r}$ and some positive real constants $q, q_{1}$ and $q_{2} ; \mathbf{q}_{0}$ is a $n$-real vector such that $\left[\begin{array}{cc}P & \mathbf{q}_{\mathbf{0}} / 2 \\ \mathbf{q}_{0}^{T} / 2 & q_{1}\end{array}\right]>0$. The subsequent result is proved in Appendix B.

Theorem 3. Assume that there exist real constants $K_{1} ; K_{2} \geqslant K_{1}$ such that $K_{1} \leqslant$ $\frac{k(t)}{1+d k(t)} \leqslant K_{2}<\infty$ for all $t \geqslant 0$. Thus, the system (17) is absolutely stable on $[0, \infty)$ independent of the delays, for any nonlinearity within the given class, provided that 
1. There exist real $n$-matrices $P=P^{T}>0, S_{i}=S_{i}^{T}>0$ for $i=\overline{1, r}$, some positive real constants $\varepsilon, q, q_{1}$ and $q_{2} ;$ and $\mathbf{q}_{\mathbf{0}} \in \mathbf{R}^{\mathbf{n}}, w \in \mathbf{R}, l \in \mathbf{R}^{\mathbf{n}}$ such that $\left[\begin{array}{cc}P & \mathbf{q}_{\mathbf{0}} / 2 \\ \mathbf{q}_{0}^{T} / 2 & q_{1}\end{array}\right]>0$.

2. The constraints (3a), (3b), and

$$
\begin{gathered}
\left(A^{T}+\varepsilon I\right) P+P(A+\varepsilon I)+\sum_{i=1}^{r} S_{i} \leqslant-l l^{T}-\frac{\left|k_{2}\right|}{1-d\left|k_{2}\right|} g A c c^{T} A \\
\bar{Q}^{\prime}(t)=\left[\begin{array}{ccccc}
-\varepsilon P & P A_{1} & \ldots & P A_{r} & m(t) \\
A_{1}^{T} P & -S_{1} & 0 & q A_{1}^{T} c \\
: & & : & & \vdots \\
A_{r}^{T} P & 0 & & -S_{r} & q A_{r}^{T} c \\
m^{T}(t) & q c^{T} A_{1} & \ldots & q c^{T} A_{r} & f(t)
\end{array}\right]<-\bar{q}^{\prime} I<0
\end{gathered}
$$

for all $t \geqslant 0$ hold simultaneously for some real constant $g \geqslant 0$ if $\left|k_{2}\right|<d^{-1} ; g \leqslant 0$ if $\left|k_{2}\right| \geqslant d^{-1}$ for $d>0$; and $g \geqslant 0$ if $d=0$; where

$$
\begin{aligned}
& m(t)=P b-\frac{1}{2}\left(A^{T} \mathbf{q}_{\mathbf{o}}+q_{2} c\right)+\frac{1}{1+d k(t)}\left(\frac{c^{T} b k(t)}{2} \mathbf{q}_{\mathbf{0}}-\left(k(t) q_{1}+\frac{q}{2}\right) A^{T} c\right) \\
& f(t)=b^{T} \mathbf{q}_{\mathbf{0}}+d q_{2}+\left(2 q_{1} k(t)+q\right) \frac{c^{T} b}{1+d k(t)}
\end{aligned}
$$

A similar result holds for the Principal Case and, on the other hand, extensions to absolute stability dependent of the delays follow also directly as Theorem 2 (absolute stability dependent of the delays) extends Theorem 1 (absolute stability independent of the delays)for the system configuration (1). Both discussion are omitted in order not to overlength the paper.

\section{Examples}

EXAMPlE 1. Consider (1) with $n=2, r=1 ; b^{T}=(-1, \beta) k ; c^{T}=(1,0)$ and $A=\left[\begin{array}{cc}0 & 1 \\ -a \beta & -(\beta+a)\end{array}\right] ; A_{1}=a_{1}\left[\begin{array}{cc}0 & 1 \\ -a & -1\end{array}\right]$ with $a>0, \beta>0$ and $d>0$. The open-loop forward-loop is globally asymptotically stable for any nonlinear device of the given class, namely, the system is absolutely stable in the sector $[0, \infty)$ if $|\delta|<\left|\frac{a}{a_{1}}\right|$ and $A$ is a stability matrix satisfying $A^{T} P+P A=-L$ for some real $n$-matrix $P=P^{T}>0$ for any given real $n$-matrix $L=L^{T}>0$. It is well-known that $P=\int_{0}^{\infty} \mathrm{e}^{\mathrm{A}^{\mathrm{T}} \tau} \mathrm{Le}^{\mathrm{A} \tau} \mathrm{d} \tau$. A simple calculation yields that Theorem 1 holds with $q=\frac{P b-c}{\sqrt{d}} \delta_{0}$ being calculated by using $\alpha \leqslant \alpha_{0}\left|a a_{1}\right|$ and $\alpha_{0} \leqslant \sqrt{1+\left(\frac{(\beta+a)^{2}+(\beta+a)}{a^{2} \beta^{2}}\right)}$ obtained from the calculations of the related $H_{\infty}$-norms. Thus, from Theorem 1 , the feedback system (1) is absolutely stable for any nonlinear device (1c) or (1d), i.e., for the feed forward linear plant being either 
in the Principal Case or in the Simplest Particular Case. The associate transfer function posses a strictly stable zero/pole cancellation at $s=-\beta$ which has not been taken into account in the above calculations. This is reasonable when the transfer function numerator and denominator are not factored explicitly from the state-space description especially for high order systems. If such a cancellation is known and removed for a minimum statespace realization of (1d)-(1b) resulting in $A=-a, A_{1}=-a, b=k, c=1$ then $P=1 / k$ and $q=0$ guarantee the result for $\delta_{0}=\frac{d a^{2}}{\left|a_{1}\right|(d a+2 k(a+1) a)}$ and $|\delta| \in\left[0, \delta_{0}\right)$. In this example, the calculations may also be performed from the real part of the transfer function once the cancellation, if known, is removed. In this case, this leads to $d>0$ and $|\delta| \in\left[0, \delta_{0}\right)$ for $\delta_{0}=\frac{a}{\mid a_{1}}$ which is the weakest found constraint. In this example, the calculations may also be performed from the real part of the transfer function once the cancellation, if known, is removed. However, obtaining factored transfer functions from a state-space realization is not direct for high-order systems in the presence of delays. This fact justifies the adequacy of the proposed method to practical problems.

EXAMPLE 2. Assume that the transfer function of the linear feed forward loop (1a)-(1b) is first-order and single-delayed given by $G(s)=\frac{c b}{s+a-\delta a_{1} \mathrm{e}^{-\mathrm{hs}}}+d$. If $d>0$ and $c=p b$ for any real $p>0$ then the open-loop linear system is Lyapunov's asymptotically stable independent of the delay size $h$ if $a>0$ and $|\delta| \in\left[0, \delta_{0}\right)$ with $\delta_{0} \leqslant \frac{a}{\left|a_{1}\right|}$ from Theorem 1 . Furthermore, the system (1) is absolutely stable in $[0, \infty)$ since Theorem 2 hold $s$ with $q=0$ since

$$
\operatorname{Re} G(j \omega)=\frac{p b^{2}\left(a-\delta a_{1} \cos \omega h\right)}{\left[\left(a-\delta a_{1} \cos \omega h\right)^{2}+\left(\omega+\delta a_{1} \sin \omega h\right)\right]^{1 / 2}}+d>0,
$$

for all frequency $\omega$. Since the transfer function of the time-invariant forward loop is positive real then the closed-loop configuration (1a)-(1c) is, in addition, asymptotically hyperstable (Sen, 1986; 2002), i.e., the nonlinear function may be even time-varying while satisfying a Popov's-type integral inequality $\int_{0}^{t} y(\tau) \Phi(y(\tau)) \mathrm{d} \tau \geqslant-\gamma_{0}^{2}$ for all time and the closed-loop system is globally Lyapunov's stable Assume that in this example $c \neq p b$ but $d>\left|\frac{c b}{a-\delta_{0} \mid a_{1}}\right|$. Thus, asymptotic hyperstability; i.e., global Lyapunov's stability of (1) follows even if the nonlinear function defining the control law (1c) or (1d) is timevarying satisfying the above integral inequality (Bergen, 1967; Sen, 1986; Gregor, 1996; Sen, 1998; 2002; Popov and Halanay, 1963). Thus, its absolute stability in the sector [0, $\infty)$ holds as well as a particular result.

EXAMPLE 3. Assume that the transfer function of (1a)-(1b) is a second-order one in the Simplest Particular Case and single-delayed given by $G(s)=\frac{c b}{s\left(s+a_{1}-\delta \mathrm{e}^{-\mathrm{hs}}\right)}+\frac{d}{s}$. Thus, the closed-loop system (1a)-(1b), (1d) is absolutely stable in $(0, \infty)$ from Theorem 1 with $\mathrm{q}>0$ if $|\delta| \in\left[0, \delta_{0}\right)$ with $\delta_{0} \leqslant\left|a_{1}\right|=\lambda a<a$ with a $>0$ and $0 \leqslant \lambda<1$ (i.e., a is an absolute upper-bound of $\left.a_{1}\right)$ provided that $d>\frac{(\lambda-1) a+q \lambda^{2} a^{2} / 4}{(1+\lambda)^{2} a^{2}}$. 


\section{Conclusions}

This paper has dealt with the absolute stability both independent and dependent of the delays of single-input single-output feedback systems consisting of time-invariant linear forward blocks including delayed dynamics with internal point delays subject to a wide class of feedback nonlinearities subject to a class of sector's-type constraints. A class of time-differentiable nonlinearities possessing time-derivative for all time while not being restricted to such a sector has also been considered. The methodology used to derive the sufficiency-type conditions for absolute stability is based on Lyapunov's second method through obtaining appropriate Lyapunov's functions.

\section{Acknowledgments}

The author is very grateful to MCYT by its partial support of this work through Project DPI 2000-0244.

\section{Appendix A}

Proof of Theorem 1. Direct calculations yield:

$$
\begin{aligned}
\dot{V}\left(x_{t}\right)= & \dot{x}^{T}(t) P x(t)+x^{T}(t) P \dot{x}(t)+\sum_{i=1}^{r}\left(x^{T}(t) S_{i} x(t)-x^{T}\left(t-h_{i}\right) S_{i} x\left(t-h_{i}\right)\right) \\
& -2 d \xi(t) \Phi(t)-2 d \xi(t) \sum_{i=1}^{r} \Phi\left(t-h_{i}\right)+2 q \Phi(t) \dot{y}(t) \\
& +d \sum_{i=1}^{r} q_{i}\left(\Phi^{2}(t)-\Phi^{2}\left(t-h_{i}\right)\right)
\end{aligned}
$$

where $\Phi(t)$ denotes simply the implicit function of time $\Phi(y(t))$. The substitution of $\dot{y}(t)$ from (1c) via (1a)-(1b) and $\dot{x}(t)$ from (1a) into (A.1) yields after grouping terms and using $d \xi(t)=y(t)-c^{T} x(t)$ :

$$
\begin{aligned}
& \dot{V}\left(x_{t}\right)=x^{T}(t)\left(A^{T} P+P A+\sum_{i=1}^{r} S_{i}\right) x(t)+2 \sum_{i=1}^{r} x^{T}(t) P A_{i} x\left(t-h_{i}\right) \\
& \quad-\sum_{i=1}^{r} x^{T}\left(t-h_{i}\right) S_{i} x\left(t-h_{i}\right)-2 \Phi(t) b^{T} P x(t)-2 x^{T}(t) P b \sum_{i=1}^{r} k_{i} \Phi\left(t-h_{i}\right) \\
& +2 \Phi(t) c^{T}(I+q A) x(t)-2 q\left(d+c^{T} b\right) \Phi^{2}(t)-2 y(t) \Phi(t) \\
& +2 c^{T} x(t) \sum_{i=1}^{r} k_{i} \Phi\left(t-h_{i}\right)+2 q \Phi(t) c^{T} \sum_{i=1}^{r} A_{i} x\left(t-h_{i}\right)
\end{aligned}
$$




$$
\begin{aligned}
& -2 q \Phi(t) c^{T} b \sum_{i=1}^{r} k_{i} \Phi\left(t-h_{i}\right)-2 q \mathrm{~d} \Phi(t) \sum_{i=1}^{r} k_{i} \Phi\left(t-h_{i}\right) \\
& +\sum_{i=1}^{r} q_{i}\left(\Phi^{2}(t)-\Phi^{2}\left(t-h_{i}\right)\right) \\
\leqslant & -\varepsilon x^{T}(t) P x(t)-(l x(t)-w \Phi(t))^{2}-q\left(d+c^{T} b\right) \Phi^{2}(t)-2 \Phi(t) y(t) \\
& -\bar{x}^{T}(t) \Delta Q^{\prime} \bar{x}(t) \leqslant \bar{x}^{T}(t) Q \bar{x}(t)<0 \quad \text { for } \bar{x}(t) \neq 0,
\end{aligned}
$$

from (3a)-(3c) and (4), since $Q<0$, where:

$$
\bar{x}(t)=\left(x^{T}(t), x^{T}\left(t-h_{1}\right), \ldots, x^{T}\left(t-h_{r}\right), \Phi(t), \Phi\left(t-h_{2}\right), . ., \Phi\left(t-h_{r}\right)\right)^{T} .
$$

Thus, $\|\bar{x}(t)\|$ is bounded on $[0, \infty)$ and converge asymptotically to zero as time tends to infinity for all $\Phi \in[0, \infty)$ if $t \geqslant 0$. Thus, $\mathrm{x}(\mathrm{t})$ and $u(t)=\dot{\xi}(t) \rightarrow 0$ as $t \rightarrow \infty$ since $\Phi(y(t)) \rightarrow 0$. Thus, $y(t) \rightarrow 0$ as $t \rightarrow \infty$. If $d>0$ then, in addition, $\xi(t) \rightarrow 0$ from (1c) as $t \rightarrow \infty$.

Outline of Proof of Corollary 1. The set of constraints (1) is a particular case of the conditions of Theorem 1 for $d=0$. Thus, $\|x(t)\|$ is bounded and converges asymptotically to zero as time tends to infinity. Also, $y(t)$ is bounded on $[0, \infty)$ and $y(t) \rightarrow$ $d \xi(t) \rightarrow 0 \Rightarrow u(t) \rightarrow 0$ as $t \rightarrow \infty$ since $d=0$. For the set of constraints (2), the particular Lyapunov's function candidate obtained from (2) with $w=q=q_{i}=0, i=\overline{1, r}$ and $l=0$ is also a particular Lyapunov's function of time-derivative satisfying:

$$
\begin{aligned}
\dot{V}\left(x_{t}\right) \leqslant & \left(x^{T}(t), x^{T}\left(t-h_{1}\right), \ldots, x^{T}\left(t-h_{r}\right)\right)^{T} \\
& \times Q^{\prime}\left(x^{T}(t), x^{T}\left(t-h_{1}\right), \ldots, x^{T}\left(t-h_{r}\right)\right)^{T} \leqslant 0,
\end{aligned}
$$

and nonzero for $x(t) \neq 0$. The proof follows directly as a result.

\section{Appendix B}

Proof of Theorem 3. Direct calculations to obtain and upper-bound the time-derivative of the Lyapunov's function candidate (20) using (17)-(19) and (21)-(23) yield directly:

$$
\begin{aligned}
\dot{V}\left(x_{t}\right)= & 2\left[\dot{x}^{T}(t) P x(t)+q_{1} \Phi(t) \dot{\Phi}(t)\right]+\dot{x}^{T}(t) \mathbf{q}_{0} \Phi(t)+x^{T}(t) \mathbf{q}_{\mathbf{0}} \dot{\Phi}(t) \\
& +q_{2} \Phi(t) y(t)+q \Phi(t) \dot{y}(t)-2 d \xi(t) \\
= & \left(x^{T}(t) A^{T}-b^{T} \Phi(t)-\sum_{i=1}^{r} x^{T}\left(t-h_{i}\right) A_{i}^{T}\right) P x(t) \\
& +x^{T}(t) P\left(A x(t)-b \Phi(t)-\sum_{i=1}^{r} A_{i} x\left(t-h_{i}\right)\right)
\end{aligned}
$$




$$
\begin{aligned}
& +\left(x^{T}(t) A^{T}-b^{T} \Phi(t)-\sum_{i=1}^{r} x^{T}\left(t-h_{i}\right) A_{i}^{T}\right) \mathbf{q}_{0} \Phi(t) \\
& +x^{T}(t) \mathbf{q}_{0} \frac{k(t) c^{T}}{1+d k(t)}\left(A x(t)-b \Phi(t)-\sum_{i=1}^{r} A_{i} x\left(t-h_{i}\right)\right) \\
& +2 q_{1} \Phi(t) \frac{k(t) c^{T}}{1+d k(t)}\left(A x(t)-b \Phi(t)-\sum_{i=1}^{r} A_{i} x\left(t-h_{i}\right)\right) \\
& +q_{2} \Phi(t)\left(c^{T} x(t)-d \Phi(t)\right) \\
& +q \Phi(t) \frac{c^{T}}{1+d k(t)}\left(A x(t)-b \Phi(t)-\sum_{i=1}^{r} A_{i} x\left(t-h_{i}\right)\right) \\
& \leqslant \bar{x}^{\prime T}(t) \bar{Q}^{\prime \prime}(t) \bar{x}^{\prime}(t)<0 \quad \text { if } \bar{x}^{\prime}(t) \neq 0,
\end{aligned}
$$

where the $n$-matrix function $\bar{Q}^{\prime \prime}(t) \leqslant-\bar{q}^{\prime \prime} I\left(\bar{q}^{\prime \prime}>0\right)$, since $\bar{Q}^{\prime}(t) \leqslant-\bar{q}^{\prime} I\left(\bar{q}^{\prime}>0\right)$, is identical to $\bar{Q}^{\prime}(t)$ in $(23 a)$ except for the $(1,1)$ matrix block which is now given by

$$
A^{T} P+P A+\frac{q_{0} k(t) c^{T} A}{1+d k(t)}+\sum_{i=1}^{r} S_{i}<0
$$

and $\bar{x}^{\prime}(t)=\left(x^{T}(t), x^{T}\left(t-h_{1}\right), \ldots, x^{T}\left(t-h_{r}\right), \Phi(t)\right)^{T}$. Thus, the proof follows directly from (B.2) since the Lyapunov's function candidate (B.1) is proved to be a Lyapunov's function from (B.2).

\section{References}

Barnett, S., and R.G. Cameron (1985). Introduction to Mathematical Control Theory. 2nd Edition, Oxford University Press, Oxford, UK (1990).

Bergen, A.R. (1967). A method for general design of positive real functions. IEEE Trans. Autom. Control, AC-12 (7), 764-769.

Gorecki, V., S. Fuska, P. Grabowski and A. Korytowski (1989). Analysis and Synthesis of Time-Delay Systems. John Wiley and Sons, Varszawa.

Gregor, J. (1996). On the design of positive real functions. IEEE Trans. Circuits and Systems, CIS-43 (11), 945-947.

Hsiao, J.M., and C.C. Cheng (2002). Robust tracking controllers for perturbed interconnected systems. In Preprints of the IFAC 15th Triennial World Conference. Barcelona.

Kailath, T. (1980). Linear Systems. Prentice-Hall, Englewood Cliffs, N.J.

Niculescu, S.I. (2001). Delay effects on stability. A robust control approach. In M. Thoma and M. Morari (Eds.), Lecture Notes in Control and Information, 269. Springer-Verlag, Berlin.

Popov, V.M., and A. Halanay (1963). On the stability of nonlinear automatic control systems with lagging argument. Autom. Remote Control, 23, 783-786.

Sen, M. de la (1986). Stability of composite systems with an asymptotically hyperstable block. Int. J. of Control, 44 (6), 1769-1775.

Sen, M. de la (1998). A method for general design of positive real functions. IEEE Trans. Circuits and Systems, CIS-45 (7), 312-314

Sen, M. de la (2002). Preserving positive realness through discretization. J. of Positivity, 6 (1), 31-45, Kluwer Academic Publishers.

Vidyasagar, M. (1993). Nonlinear Systems Analysis. Prentice-Hall, Englewood Cliffs, New Jersey, 2nd Edition. 
M. de la Sen was born in Arrigorriaga, Bizkaia, Spain in 1953. He received his MSc degree with honors in applied physics from the University of the Basque Country, his PhD degree also in applied physics from the same university in 1979, and the degree of "Docteur d'Etat-ès-Sciences Physiques" (specialité Automatique et Traiment du Signal) with "mention très honorable" in 1987. He has had several teaching positions in the University of the Basque Country at Bilbao, Spain, where he is currently professor of systems and control engineering. He has also had the positions of visiting professor in the University of Grenoble, France and the University of Newcastle, New South Wales, Australia. He has authored or co-authored a number of papers in the fields of adaptive systems, discrete systems, ordinary and functional (time-delay) differential equations, and mathematical systems theory. He had supervised fifteen doctoral thesis and a number of master research works in those fields. 


\section{Vieno ięjimo vieno išèjimo su pastoviais vidiniais laiko vẻlinimais} sistemu absoliutusis stabilumas

\section{Manuel de la SEN}

Straipsnyje nagrinèjamas vieno ięjimo vieno išèjimo sistemu su laiko vèlinimais absoliutusis stabilumas, kai baigtinis skaičius sistemos vidinių vẻlinimų, tenkinančių tam tikras nelygybes, yra pirmame ir trečiame kvadratuose. Rezultatai gauti naudojant Liapunovo funkcijas ir pagrịsti Liapunovo stabilumo analize. Sistemų stabilumo analizė atlikta, kai sistemoje vèlinimai yra ir kai ju nèra. 